The Dissection of any two Triangles into mutually similar pairs of Triangles.

\author{
By R. F. Muirhead, M.A., B.Sc.
}

1. The problem discussed is that of dissecting two given triangles into triangular parts which shall consist of mutually similar pairs of triangles, so that the first given triangle $A$ being dissected into the triangles $a_{1}, a_{2}, a_{3} \ldots$, and the second given triangle $\mathrm{B}$ being dissected into the triangles $b_{1}, b_{2}, b_{3} \ldots$, we shall have $a_{1}$ similar to $b_{1}, a_{2}$ to $b_{2}$, and so on.

2. A little consideration will show that in general the problem is insoluble, if the number of parts of each given triangle is restricted to two.

We shall therefore consider the case in which each of the two given triangles is to be dissected into three triangular parts.

3. The first type of solution here given, is when an internal point of each triangle is joined to each of its vertices, so as to divide it into three triangular parts. The solution is as follows :-

Figure 1.

Let $\mathrm{ABC}$ be one of the given triangles.

On $\mathrm{BC}$, on the side opposite from $\mathrm{ABC}$, describe the triangle $\mathrm{DBC}$ similar to the other given triangle $A^{\prime} B^{\prime} C^{\prime}$, and join $A D$.

Make $\angle \mathrm{BAE}=\angle \mathrm{CAD}$

and $\angle \mathrm{ABE}=\angle \mathrm{ADC}$.

Join CE.

Make $\angle \mathrm{BDF}=\angle \mathrm{CDA}$

and $\angle \mathrm{DBF}=\angle \mathrm{DAC}$.

Join CF.

By construction, the triangle $\mathrm{ABE}$ is equiangular with the triangle ADC. $\quad \therefore$ BA : AE : : DA : AC.

$$
\therefore \text { BA : AD : : EA : AC. }
$$

Hence, $\angle \mathrm{BAD}$ being $=\angle \mathrm{EAC}$, the triangles $\mathrm{BAD}, \mathrm{EAC}$ are also similar to one another. 
In like manner, the triangles DFB, DFC are similar to DCA and DBA respectively.

Hence DFB is similar to BEA, and DFC to CEA.

It follows at once that $\angle \mathrm{BEO}=\angle \mathrm{BFC}$.

Again BE: EC is compounded of BE:EA and EA:EC, i.e., of $\mathrm{DF}: \mathrm{FB}$ and $\mathrm{CF}: \mathrm{FD}, \therefore$ it is the same as $\mathrm{CF}: \mathrm{FB}$.

Hence the triangle BEC is similar to the triangle CFB, and in fact the figure BECF is a parallelogram.

Thus AEB, BEC, CEA are similar to BFD, OFB, and DFC respectively.

4. For a given pair of triangles, the solutions of this type are 9 in number, if we distinguish between triangles which are "symmetrically" or "perversely" equal to one another; for the side of $A B C$ on which $D B C$ is to be placed can be chosen in 3 ways, and the side of $\mathrm{A}^{\prime} \mathrm{B}^{\prime} \mathrm{C}^{\prime}$ which is to be homologous to $\mathrm{BO}$ in the triangle $\mathrm{DBC}$ can also be chosen in 3 ways.

5. If, however, we drop the distinction between a plane figure and its perverse, i.e., if we permit the triangle $A^{\prime} B^{\prime} C^{\prime}$ to be turned over on its plane, there will be 18 solutions of the type we are considering.

6. It is to be noted, however, that some of the solutions may involve negative areas. This will happen when the sum of the angles $A$ and $D$ is greater than two right angles. The points $E$ and $F$ will then lie without the triangles $A B C, D B C$, respectively; and the problem solved by the construction will in that case be this: To find two pairs of similar triangles such that one of each pair being taken, they will build up a quadrilateral, and the remaining two will also build up a quadrilateral, these quadrilaterals being decomposable into two triangles of given shape and two others which are similar to one another.

7. Note also that if $A B C D$ be a cyclic quadrilateral, the triangles $\mathrm{BEC}, \mathrm{BFC}$ collapse, so that the given triangles in that case can be dissected into two pairs of mutually similar triangles.

8. The second type of solution here considered is when one triangle $\mathrm{ABC}$ is dissected by joining an angular point $A$ to a 
point $D$ of the opposite side $B C$, then joining enother angular point $O$ to a point $E$ in the line $A D$, and the second given triangle is dissected in like manner. The solution may be stated thus :-

\section{Fradre 2.}

Let $A B C$ and $A^{\prime} B^{\prime} C^{\prime}$ be the two given triangles.

Let us make $C^{\prime} A^{\prime} D^{\prime}=B$.

$$
\therefore \quad A^{\prime} D^{\prime} B^{\prime}=B+C^{\prime} \quad \text { and } \quad B^{\prime} A^{\prime} D^{\prime}=A^{\prime}-B \text {. }
$$

Make $\quad \mathrm{CAD}=\mathbf{A}^{\prime}-\mathrm{B}$, then $\mathrm{DAB}=\mathrm{A}-\mathbf{A}^{\prime}+\mathbf{B}$.

Make $\quad A^{\prime} C^{\prime} E^{\prime}=A-A^{\prime}+B, \quad \therefore \quad D^{\prime} C^{\prime} E^{\prime}=C^{\prime}-A+A^{\prime}-B$

$$
=\mathbf{C}-\mathbf{B}^{\prime} \text {. }
$$

Make $\quad \mathbf{A C E}=\mathrm{B}^{\prime}, \therefore \quad \mathrm{DCE}=\mathbf{C}-\mathrm{B}^{\prime}=\mathbf{D}^{\prime} \mathbf{C}^{\prime} \mathbf{E}^{\prime}$.

And $\quad E D C=B+A-A^{\prime}+B=D^{\prime} \mathbf{E}^{\prime} C^{\prime}$

$$
=\mathrm{B}-\mathrm{C}+\mathrm{C}^{\prime}+\mathrm{B}^{\prime} \text {. }
$$

Thus the triangles $\mathrm{ABD}, \mathrm{ACE}, \mathrm{CDE}$ are similar to the triangles $\mathrm{C}^{\prime} \mathrm{A}^{\prime} \mathrm{E}^{\prime}, \mathrm{A}^{\prime} \mathrm{B}^{\prime} \mathrm{D}^{\prime}, \mathrm{C}^{\prime} \mathbf{E}^{\prime} \mathrm{D}^{\prime}$, respectively; the homologous sides being given in the same order in the two sets. similar.

We may note, however, that two of the pairs are perversely

9. The question may be put: How many solutions of this type exist for the dissection of a given pair of triangles? Noting that the undivided angles ( $B$ and $B^{\prime}$ in the above dissection) may be chosen in 9 ways from the two given triangles, and that there is then a further choice of $2 \times 2$ ways of completing the construction, we see that there would seem to be 36 solutions of this type. But of these some would involve negative areas: if, e.g., the angle $B$ were greater than the angle $A^{\prime}$ in the solution above given, $A D^{\prime}$ would fall outside the triangle $A^{\prime} B^{\prime} C^{\prime}$, as in

\section{Figure 3.}

The problem solved in this figure would be: "Given two triangles, to form two triangles similar to one another, each made from one of the given triangles and one of each of two other pairs of similar triangles." Thus $\mathrm{ACE}$ and $\mathrm{AB}^{\prime} \mathrm{D}^{\prime}$ are similar to one another, also $\mathrm{ODE}$ and $\mathrm{C}^{\prime} \mathrm{E}^{\prime} \mathrm{D}^{\prime}$, and then together with the given triangles $A B C, A^{\prime} B^{\prime} C^{\prime}$ make up the similar triangles $A B D$ and $\mathbf{C}^{\prime} \mathbf{A}^{\prime} \mathbf{E}^{\prime}$. 
Out of the 36 theoretically possible solutions of the original problem, the number in which no "negative areas" occur would depend on the shapes of the given triangles, and I do not here attempt to discuss that point any further.

10. Lastly, it may be shown that solutions of the two types that have been discussed, are particular cases of a more general dissection which dissects two triangles into two sets of four triangles which are similar in pairs, one angle in the figure being arbitrary.

\section{Figure 4.}

Take $A B C D$ as in the first case.

Draw $\mathrm{AE}^{\prime} \mathrm{E}^{\prime \prime}$ making $\angle \mathrm{BAE}$ arbitrary, $=\theta$ say.

Make $\angle \mathrm{DBF}=\angle \mathrm{BAE}^{\prime}$,

$\angle \mathrm{DCF}^{\prime}=\angle \mathrm{CAE}^{\prime}$,

$\angle \mathrm{BCE}=\angle \mathrm{CBF}$,

$\angle \mathrm{CBE}=\angle \mathrm{BCF}$,

and $\angle \mathrm{BDF}^{\prime}=\angle \mathrm{ABE}$;

where $\mathrm{AE}^{\prime} \mathrm{E}^{\prime \prime}, \mathrm{BE}^{\prime} \mathrm{E}, \mathrm{CE} \mathbf{E}^{\prime}$ meet one another in $\mathrm{E}, \mathrm{E}^{\prime}, \mathbf{E}^{\prime \prime}$,

and $\mathrm{DF}^{\prime \prime} \mathrm{F}^{\prime}, \mathrm{BFF}^{\prime \prime}, \mathrm{CF}^{\prime} \mathrm{F}$ meet one another in $\mathrm{F}, \mathrm{F}^{\prime}, \mathrm{F}^{\prime \prime}$.

Then $\angle \mathrm{CDF}^{\circ}=\angle \mathrm{ACE}$, since the former, with 5 other angles, makes up the angles of the triangle BDC, while the latter, with 5 angles equal respectively to the other 5 , makes up the angles of the triangle $\mathrm{ABC}$.

Hence the triangles $\mathrm{ABE}^{\prime \prime}, \mathrm{BCE}, \mathrm{CAE}^{\prime}$ are directly similar respectively to the triangles $\mathrm{BDF}^{\prime \prime}, \mathrm{CBF}, \mathrm{DCF}^{\prime}$. Hence obviously the triangle $\mathbf{E E}^{\prime} \mathbf{E}^{\prime \prime}$ is similar perversely to the triangle $\mathrm{FF}^{\prime} \mathrm{F}^{\prime \prime}$. Thus the more general problem is solved.

11. Now if we take $\theta=0$, the triangles $A B E^{\prime \prime}$ and $B D F^{\prime \prime}$ will collapse, giving us a solution of the second type by dissection into three parts. The same result may also be obtained by choosing $\theta$ so that one of the other angles shall vanish, such as CBE, or BCE'.

12. Again if we choose $\theta$ so that the triangle $\mathrm{EE}^{\prime} \mathrm{E}^{\prime \prime}$ shall shrink to a point, the trinangle $\mathrm{FF}^{\prime} \mathrm{F}^{\prime \prime}$ will do so also, and we shall have a solution of the first type. 
13. Certain properties of the Figure 4 may be noted. Taking $B^{\prime}, C^{\prime}, A^{\prime}$ to denote the angles of the triangle $B C D$,

$$
\begin{aligned}
& \angle \mathbf{B A E}=\angle \mathrm{DBF}=\theta ; \\
& \angle \mathbf{E}^{\prime} \mathbf{A C}=\angle \mathrm{DCF}^{\prime}=\mathbf{A}-\theta ; \\
& \angle \mathrm{ACE}=\angle \mathrm{CDF}^{\prime}=\mathbf{C}-\mathbf{B}^{\prime}+\theta ; \\
& \angle \mathrm{ECB}=\angle \mathbf{C B F}=\mathbf{B}^{\prime}-\theta ; \\
& \angle \mathrm{CBE}=\angle \mathrm{BCF}=\mathbf{C}^{\prime}-\mathbf{A}+\theta ; \\
& \angle \mathrm{EBA}=\angle \mathbf{B D F}^{\prime}=\mathbf{B}+\mathbf{A}-\mathbf{C}^{\prime}-\theta=\mathbf{B}^{\prime}+\mathbf{A}^{\prime}-\mathbf{C}-\theta .
\end{aligned}
$$

The exterior angles of the triangle $\mathbf{E E}^{\prime} \mathbf{E}^{\prime \prime}$ are $A+\mathbf{A}^{\prime}, \mathbf{B}+\mathbf{B}^{\prime}, \mathbf{C}+\mathbf{C}^{\prime}$, respectively, so that the shape of the triangles $\mathrm{EE}^{\prime} \mathrm{E}^{\prime \prime}$ and $\mathrm{FF}^{\prime} \mathrm{F}^{\prime \prime}$ is independent of $\theta$.

The triangles $\mathrm{BCE}, \mathrm{CBF}$ are equal. The triangles $\mathrm{BD} \mathrm{F}^{\prime \prime}, \mathrm{ABE}^{\prime \prime}$ are in the duplicate ratio of $\mathrm{D} \mathrm{B}: \mathrm{B} \mathbf{A}$.

$$
\begin{aligned}
& \text { " " } \quad \text { CDF", } \mathrm{CAE}^{\prime} " \text { " " " " } \quad \text { DC:CA. } \\
& \text { " " } \quad \text { EE' } \mathbf{E}^{\prime}, \mathbf{F F}^{\prime} \mathbf{F}^{\prime \prime}, \quad, \quad, \quad, \quad, \quad \text {, } \mathbf{E}^{\prime} \mathbf{E}^{\prime \prime}: \mathbf{F}^{\prime} \mathbf{F}^{\prime \prime} \text {. }
\end{aligned}
$$

The ratio $\mathrm{E}^{\prime} \mathrm{E}^{\prime \prime}: \mathrm{F}^{\prime} \mathrm{F}^{\prime \prime}$ is expressible as a somewhat complicated function of $\theta$ and the angles of the two triangles.

14. We might discuss the problem algebraically so far as the angles of the parts are concerned, as follows:-

In a solution of the first type there are two degrees of freedom in the position of $\mathbf{E}$, and two in the position of $\mathrm{F}$. Taking $\theta, \phi, \theta^{\prime}, \phi^{\prime}$, to denote the angles BAE, EBC, BDF, FBC respectively, it is clear that, $\mathbf{E}$ and $\mathbf{F}$ being any points in the given triangles, $\theta, \phi, \theta^{\prime}, \phi^{\prime}$ are aribitrary, and when chosen, determine the positions of $E$ and $F$ and therefore also the angles BCE, etc. Now to secure that the triangular segments shall be similar in pairs, we must for each pair equate two angles of one respectively to two angles of the other. Thus on the whole we have six equations to satisfy, by means of the four variables $\theta, \phi, \theta^{\prime}, \phi^{\prime}$. The fact that there is a general solution would seem to indicate that these equations are not all independent: but $I$ have not further considered this rather curious point, nor have I attempted to classify or discuss the great variety of ways in which the six equations can be written down, nor to determine whether the solutions I have given are the only possible ones.

Similar remarks apply to solutions of the second type. 
15. The analogy of the problem here discussed to that of the dissection of figures into mutually congrvent parts will no doubt have been noticed. Of course any dissection by which two equal triangles are made mutually congruent will serve to solve the problem of this paper for the case of two unequal triangles. But there is a special interest in the solutions given here, on account of the small number of parts necessary. This is rendered possible by the fact that there is an additional element of freedom in the problem, as compared with that which would make the ratio of each part of one triangle to the corresponding part of the other the same as that of the first whole to the second.

In Fig. 1 the angles BEC, CEA, AEB are respectively equal to $A+A^{\prime}, B+B^{\prime}, C+C^{\prime}$; and this, as $\mathrm{Mr}$ John Dougall has pointed out to me, enables us to construct the point $E$ by means of segments of circles containing these angles, described on the sides of ABC. This construction is more symmetrical. Interesting trigonometrical problems might be based on it. 